\title{
Real-Time Tsunami Forecasting: Challenges and Solutions
}

\author{
Vasily V. Titov* \\ University of Washington, Joint Institute for the Study of the Atmosphere and \\ Ocean (JISA0), Box 354235 Seattle, WA 98195-4235, U.S.A \\ F. I. González, E. N. Bernard, M. C. Eble and H. O. Mofjeld \\ NOAA/Pacific Marine Environmental Laboratory (PMEL), 7600 Sand Point Way \\ NE, Seattle, WA 98115-6349, U.S.A.
}

\section{J. C. Newman and A. J. Venturato \\ University of Washington, Joint Institute for the Study of the Atmosphere and Ocean (JISA0), Box 354235 Seattle, WA 98195-4235, U.S.A}

25 September 2003

\begin{abstract}
A new method for real-time tsunami forecasting will provide NOAA's Tsunami Warning Centers with forecast guidance tools during an actual tsunami event. PMEL has developed the methodology of combining real-time data from tsunameters with numerical model estimates to provide site- and event-specific forecasts for tsunamis in real time. An overview of the technique and testing of this methodology is presented.
\end{abstract}

\section{Keywords:}

Abbreviations: BPR - bottom pressure recorder; DARPA - Defense Advanced Research Projects Agency; MOST - Method of Splitting Tsunamis; PMEL - Pacific Marine Environmental Laboratory; NASA - National Aeronautics and Space Administration; NHTMP - National Tsunami Hazard Mitigation Program; NOAA National Oceanic and Atmospheric Administration; SIFT - Short-term Inundation Forecasting; TWC - Tsunami Warning Center

\section{Background}

The 21 January 2003 Workshop on Far-field Tsunami Forecast Guidance recommended development and implementation of the next generation tools to provide Far-field Tsunami Forecast Guidance (González et al., 2003). Following this recommendation, the U.S. National Tsunami Hazard Mitigation Program (NTHMP) has funded the development of the tsunami forecast guidance tools for NOAA's Tsunami Warning Centers (TWCs) and emergency managers (NTHMPSG, 2003). The collaborative efforts will combine several tsunami forecast methodologies (Titov et al., 2001; Wei et al., 2003; Whitmore, 2003) into practical tsunami forecast tools and implement them at TWCs. NOAA's Pacific Marine Environmental Laboratory (PMEL) started systematic research

\footnotetext{
* Phone: (206) 526-4536, Fax: (206) 526-6485, vasily.titov@noaa.gov
} 
and development efforts to build practical tsunami forecasting tools in 1997 when the Defense Advanced Research Projects Agency (DARPA) funded the Early Detection and Forecast of Tsunami project to develop tsunami hazard mitigation tools for the Pacific Disaster Center. This work continued with follow-up grants from the Department of Defense and the National Aeronautics and Space Administration (NASA). The results of this research and prototype tools (Titov and González, 1997; Titov et al., 1999, 2001) will be the foundation for the next generation tsunami forecasting tools for the TWCs. This article provides a summary of this research and documents the accomplishments in developing the tsunami forecast tools to date.

\section{The Need for Real-Time Tsunami Forecasts}

Emergency managers and other officials are in urgent need of operational tools that will provide accurate tsunami forecast as guidance for rapid, critical decisions in which lives and property are at stake. NOAA's Tsunami Warning Centers are tasked to issue tsunami warnings for the U.S. and other nations around the Pacific. Tsunami warnings allow for immediate actions by local authorities to mitigate potentially deadly wave inundation at a coastal community. The more timely and precise the warnings are, the more effective actions can local emergency managers take and more lives and property can be saved. At present, Tsunami Warning Centers' personnel are facing a difficult challenge: to issue tsunami warning based on incomplete and ambiguous data. The initial warning decisions are based on indirect measurements of seismic waves. Tsunami confirmation by coastal tide gages may arrive too late for timely evacuation measures. This lack of information can lead to a high false alarm rate and ineffective local response to the tsunami warning. Tsunami forecasting tools based on new tsunami measurement technology and the latest modeling techniques can provide crucial additional information to guide tsunami mitigation measures in real time during tsunami propagation.

\section{Challenges of Real-Time Forecasts}

Tsunami forecasts should provide site- and event-specific information about tsunamis well before the first wave arrives at a threatened community. The only information forecasted at present is the tsunami arrival time, which is based on indirect seismic information about the

source. The next generation tsunami forecast would provide estimates 
of all critical tsunami parameters (amplitudes, inundation distances, current velocities, etc.) based on direct tsunami observations. The technical obstacles of achieving this are many, but three primary requirements are accuracy, speed, and robustness.

\subsection{ACCURACY}

Errors and uncertainties will always be present in any forecast. A practical forecast, however, minimizes the uncertainties by recognizing and reducing possible errors. In the tsunami forecast, measurement and modeling errors present formidable challenge; but advancements in the science and engineering of tsunamis have identified and researched most of them.

1. Measurement Error. Tsunami measurements are always masked by noise from a number of sources: tides, harbor resonance, instrument response, to name a few. Most of the noise can be eliminated from the record by careful consideration of its sources. However, doing it in automatic mode during real-time assessment presents a serious challenge.

2. Model Approximation Error. The physics of propagation are better understood than that of generation and inundation. For example, landslide generation physics is currently a very active research topic, and comparative studies have demonstrated significant differences in the ability of inundation models to reproduce idealized test cases and/or field observations.

3. Model Input Error. This issue is also known as the "garbage in, garbage out" problem, i.e., model accuracy can be degraded by errors in (a) the initial conditions set for the sea surface and water velocity, due to inadequate physics and/or observational information, and (b) the bathymetry/topography computational grid, due to inadequate spatial coverage, resolution, and accuracy, including the difficult issues encountered in merging data from different sources.

\subsection{SPEED}

We refer here to forecast speed, relating to the time taken to make the first forecast product available to an emergency manager for interpretation and guidance. This process involves at least two important, potentially time-consuming, steps: 
1. Data stream to TWC. Seismic wave data are generally available first, but finite time is required to interpret these signals in terms of descriptive parameters for earthquakes, landslides, and other potential source mechanisms. Tsunami waves travel much slower and time on the order of a quarter of a wave period will be needed to incorporate these data into a forecast. Seismic networks are much more dense than tsunami monitoring networks, but inversion algorithms for both are needed to provide source details.

2. Model simulation speed. Currently available computational power can provide useful, far-field, real-time forecasts before the first tsunami strikes a threatened community, if the time available for forecasting is sufficiently large, the source can be quickly specified, and an accurate computational grid is available. In fact, if powerful parallel computers and/or pre-computed model results are exploited, model execution time can be reduced almost to zero, at least in principle. In practice, of course, there will always be situations for which the source-site geometry makes it impossible to provide a warning forecast. But even a late forecast will still provide valuable assessment guidance to emergency managers responsible for critical decisions regarding response, recovery, and search-and-rescue.

\subsection{Robustness}

With lives and property at stake, reliability standards for a real-time forecasting system are understandably high; and the development of such a system is a difficult challenge. It is one thing for an experienced modeler to perform a hindcast study and obtain reasonable, reliable results. Such exercises typically take months to complete, during which multiple runs can be made with variations in the model input and/or the computational grid that are suggested by improved observations. The results are then examined for errors and reasonableness. It is quite another matter to design and develop a system that will provide reliable results in real time, without the oversight of an experienced modeler.

\section{Technology for Tsunami Forecasting}

Recent advances in tsunami measurement and numerical modeling technology can be integrated to create an optimal tsunami forecasting system. Neither technology can do the job alone. Observational networks will never be sufficiently dense because the ocean is vast. Establishing and maintaining monitoring stations is costly and difficult, 
especially in deep water. Numerical model accuracy is inherently limited by errors in bathymetry and topography and uncertainties in the generating mechanism. But combined, these techniques can provide reliable tsunami forecasts. Here, we review existing modeling and measurement tools used for the PMEL's methodology for the Short-term Inundation Forecasting (SIFT).

\subsection{Measurement}

Several real-time data sources are traditionally used for tsunami warning and forecast. They are (1) various seismic data to determine source location and some source parameters, (2) coastal tide gage data used for direct tsunami confirmation and for tsunami source inversion studies (mostly research studies not in real-time mode), and (3) new realtime deep-ocean data from the NTHMP tsunameter network (González et al., ssue). Our strategy for the real-time forecasting is to use the deepocean measurement as a primary data source for making the tsunami forecast. There are several key features of the deep-ocean data that make it indispensable for the forecast model input:

1. Rapid tsunami observation. Since tsunamis propagate with much greater speed in deeper water, the wave will reach the deep-ocean gage much sooner than the equally distant coastal gage. Therefore, a limited number of strategically placed deep-ocean gages can provide advanced tsunami observation for a large portion of the coastline. This can be illustrated by a simple consideration of the tsunami travel time difference between the tsunameter and the target coast. Consider Hilo as an example of a coastal community. Figure 1 shows contours of the difference between the tsunami travel time to Hilo and to the D125 tsunameter location for every point in the Pacific (thin lines). The thick line is the 3-hour contour, which outlines sources of tsunamis that would arrive at D125 3 hours earlier than at Hilo, leaving enough time for an evacuation decision. The 3-hour contours are also shown for other existing tsunameters (thick broken lines). The envelope of thick contours (hatched area) outlines sources of tsunamis that would be detected by at least one tsunameter in time to decide on evacuation at Hilo. This diagram demonstrates that even a sparse constellation of existing tsunameters would provide timely tsunami detection from most sources around the Pacific for Hilo (and most Hawaiian communities). Other coastal communities in the U.S. and around the Pacific are not protected as well as Hawaii by existing tsunameters - a denser coverage is required for the global tsunami forecast system. 


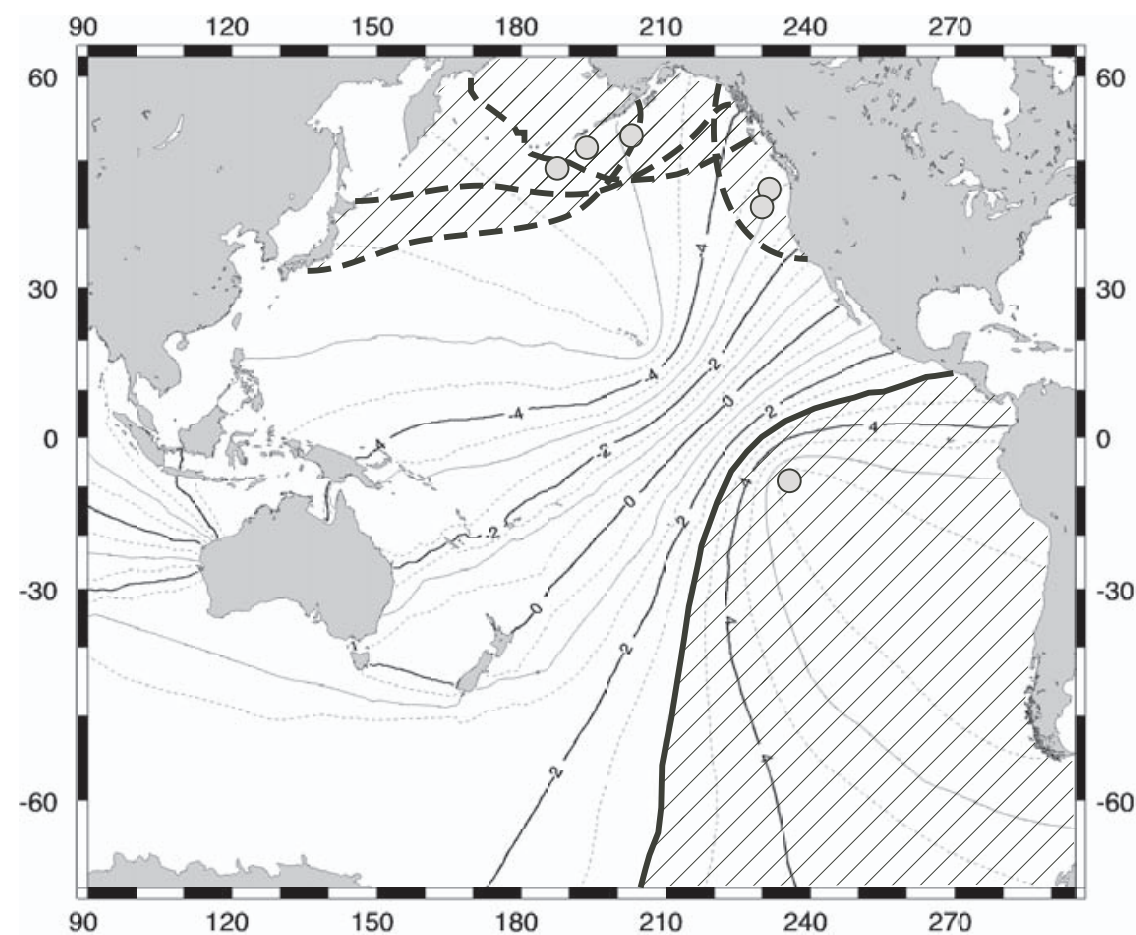

Figure 1. Contours of the time difference between the tsunami arrival at Hilo and at the D125 tsunameter station (thin lines). Thick lines show 3-hour contours of the travel time difference between Hilo and all existing tsunameters (solid line for D125, broken lines for the other tsunameters shown as gray circles). Hatched area outlines sources of tsunamis that reach at least one tsunameter 3 hours before Hilo.

Besides, a more extensive array of tsunameters would increase the warning time for each community.

2. No harbor response. Tsunameters are placed in deep water in the open ocean where a tsunami signal is not contaminated by local coastal effects. Coastal tide gages, on the other hand, are usually located inside harbors where measurements are subjected to the harbor response. As a result, only part of the tsunami frequency spectrum is accurately measured by coastal gages. In contrast, the tsunameter recording provides "unfiltered" time series with the full spectrum of the tsunami wave.

3. No instrument response. The bottom pressure recorder (BPR) of the tsunameter has a very flat and constant frequency response in the tsunami frequency range. Many coastal gages, on the contrary, have complicated and changing frequency characteristics. 
Since most of the tide gages are designed to measure tides, they do not perform well in the tsunami frequency band.

4. Linear process. The dynamic of tsunami propagation in the deep ocean is mostly linear, since amplitudes are very small compared with the wavelength. This process is relatively well understood, and numerical models of this process are very well developed. The linearity of wave dynamics also allows for application of efficient inversion schemes.

\subsection{Modeling}

The numerical modeling of tsunami dynamics has become a standard research tool in tsunami studies. Modeling methods have matured into a robust technology that has proven to be capable of accurate simulations of historical tsunamis, after careful consideration of field and instrumental historical data. NOAA's Method of Splitting Tsunamis (MOST) numerical model (Titov and González, 1997; Titov and Synolakis, 1995; 1997) is utilized for the development of the SIFT forecasting scheme. This model has been extensively tested against a number of laboratory experiments and was successfully used for many historical tsunami simulations (Bourgeois et al., 1999; Titov and Synolakis, 1995; 1996; 1997; 1998; Yeh et al., 1995). Several research groups around the world now use MOST for tsunami mitigation programs. The forecast scheme, in contrast to hindcast studies, is a two-step process where numerical models operate in different modes:

1. Data assimilation mode. The model is a part of the data assimilation scheme where it is adjusted "on-the-fly" by a real-time data stream. The model requirement in this case is similar to hindcast studies: the solution must provide the best fit to the observations. The MOST model has been tested against historical tsunamis recorded by a deep ocean BPR - the same technology as in the tsunameter system. Figure 2 shows a comparison of the simulated and measured data for the 10 June 1996 Andreanov tsunami. The good agreement confirms that the model captures the basic physics of the process and is able to reproduce the data used for the forecast.

2. Forecast mode. The model uses the simulation scenario obtained in the first step and extends the simulation to locations where measured data is not available, providing the forecast. It is difficult to fully assess the forecast potential of a particular model, since the 
quality and accuracy of the prediction will always depend on the scenario chosen by the data assimilation step. Accurate simulation of the near-shore tsunami dynamics and inundation are especially important. As a partial test of inundation forecast capability of the MOST model, the simulation of the 1993 Hokkido-Nansei-Oki tsunami has been compared with an independent dataset. The model scenario of this event is based on the field survey data (Takahashi, 1996). An independent, much denser dataset of tsunami inundation distances and heights have been obtained at PMEL from stereo photography data of Okushiri Island. Figure 3 shows a comparison of the original MOST simulation (Titov and Synolakis, 1997) with the new stereo data. Inundation values are compared for the west coast of Okushiri Island, where the highest runup was measured for this event. The MOST runup and inundation estimates compare well with both stereo and field data.

\subsection{Data Assimilation and Inversion}

An effective tsunami forecast scheme would automatically interpret incoming real-time data to develop the best model scenario that fits this data. This is a classical inversion problem, where initial conditions are determined from an approximated solution. Such problems can be successfully solved only if proper parameters of the initial conditions are established. These parameters must effectively define the solution, otherwise the inversion problem would be ill-posed.

There are several parameters that describe a tsunami source commonly used for tsunami propagation simulations. Choosing the subset of those parameters that control the deep-ocean tsunami signal is the key to developing a useful inversion scheme for tsunameter data. A sensitivity study has been conducted to explore this problem. Titov et al. $(1999,2001)$ have studied the sensitivity of far-field data to different parameters of commonly used tsunami sources. The results show that source magnitude and location define far-field tsunami signals for a wide range of subduction zone earthquakes. Other source parameters have secondary influence and can be ignored during the inversion. This result substantially reduces the size of the inversion problem for the deepocean data. An effective implementation of the inversion is achieved by using a discrete set of Green's functions to form a model source. The algorithm chooses the best fit to a given tsunameter data among a limited number of unit solution combinations by direct sorting, using a choice of misfit functions. This inversion scheme has been tested with the deep-ocean BPR records of the 1996 Andreanov tsunami. Figure 4 shows one of many tests conducted with the data. The figure shows the 

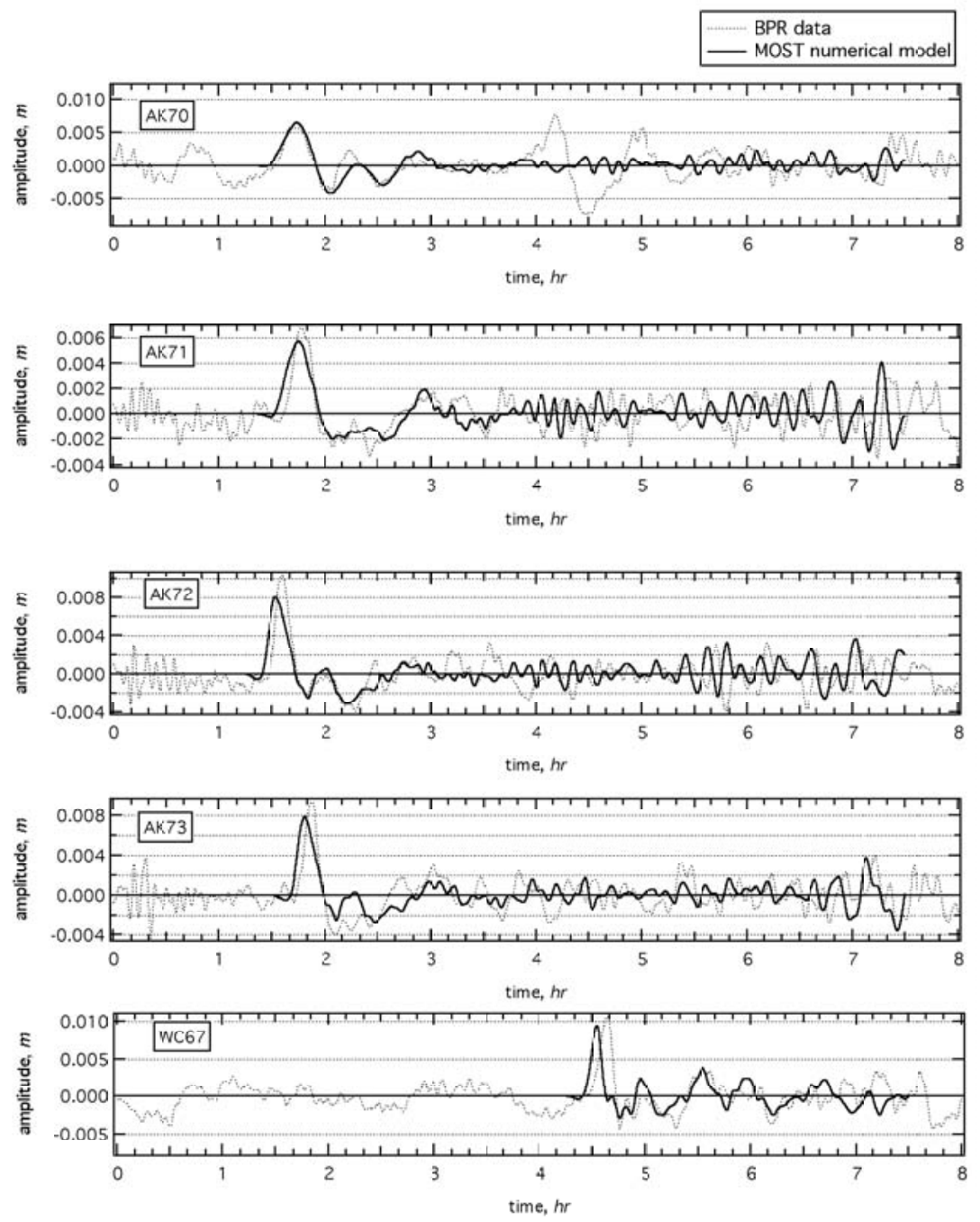

Figure 2. Comparison of the 1996 Andreanov Island tsunami propagation model (solid line) with the deep-ocean BPR data (dotted line). Locations of the BPRs are shown in Figure 4.

model scenario obtained by inverting only data from one BPR where the tsunami arrives first (AK72). Only one period of the tsunami wave record is used for the inversion. A good comparison between the model and the BPR data demonstrates the robustness of the inversion scheme. 

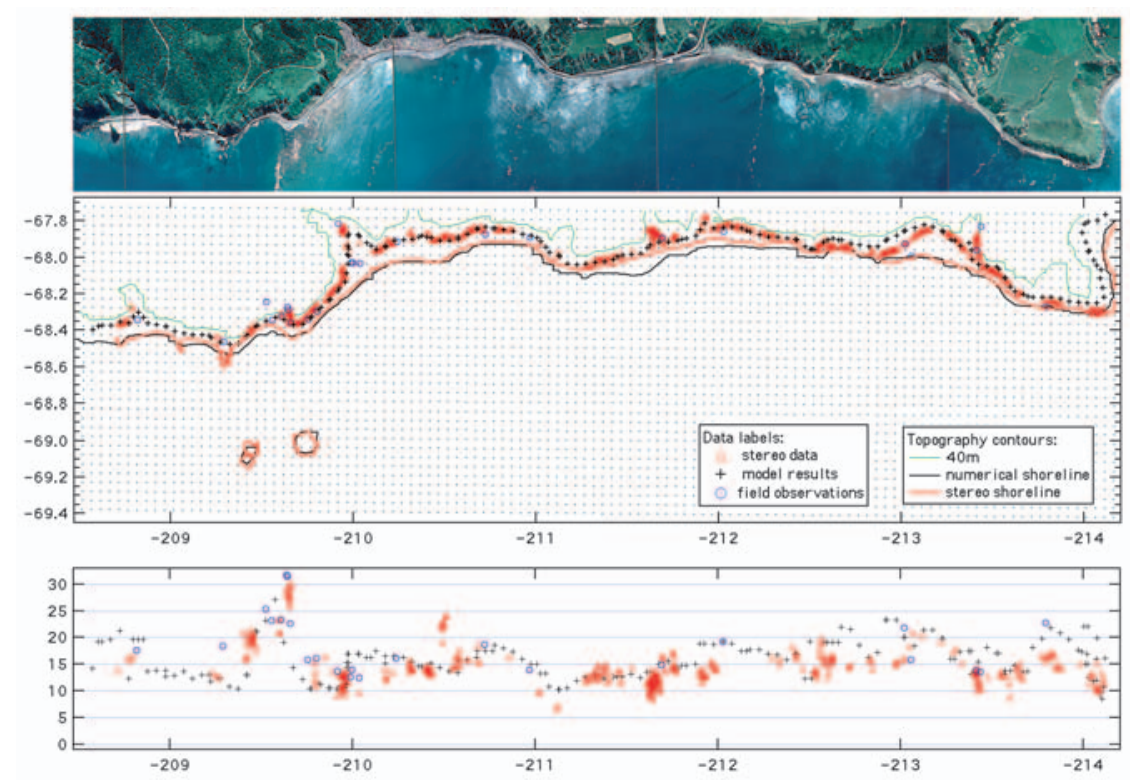

Figure 3. Comparison of the 1993 Okushiri tsunami inundation model (crosses) with field observations (circles) and stereo photo data (triangles). Top frame shows an aerial photo of the modeled area used for the stereo analysis of the inundation data. Middle frame illustrates the numerical grid used for the simulation of the same area (dots are computational nodes, contours show topography data) and compares inundation distances. Bottom frame compares maximum vertical runup for the same shoreline locations.

\section{PMEL Methodology for Tsunami Forecasting}

The previous discussion suggests that critical components of tsunami forecasting technology exist now that could provide rapid, usably accurate forecasts of a limited but important category - the first few waves of a far-field, earthquake-generated tsunami. In particular, it seems feasible to develop a forecast system that combines real-time seismic and tsunami data with a forecast database of pre-computed scenarios that have been thoroughly tested and scrutinized for reasonableness and sensitivity to errors. Later waves could also be usefully forecasted by processing real-time tsunami data with a statistical/empirical model (Mofjeld et al., 2000). Implementation of this technology requires integration of these components into a unified, robust system.

\subsection{Linear PROPAGATION MODEL DATABASE FOR UNIT SOURCES}

The source sensitivity study (Titov et al., 1999) has established that only a few source parameters are critical for the far-field tsunami characteristics, namely the location and the magnitude (assuming some 


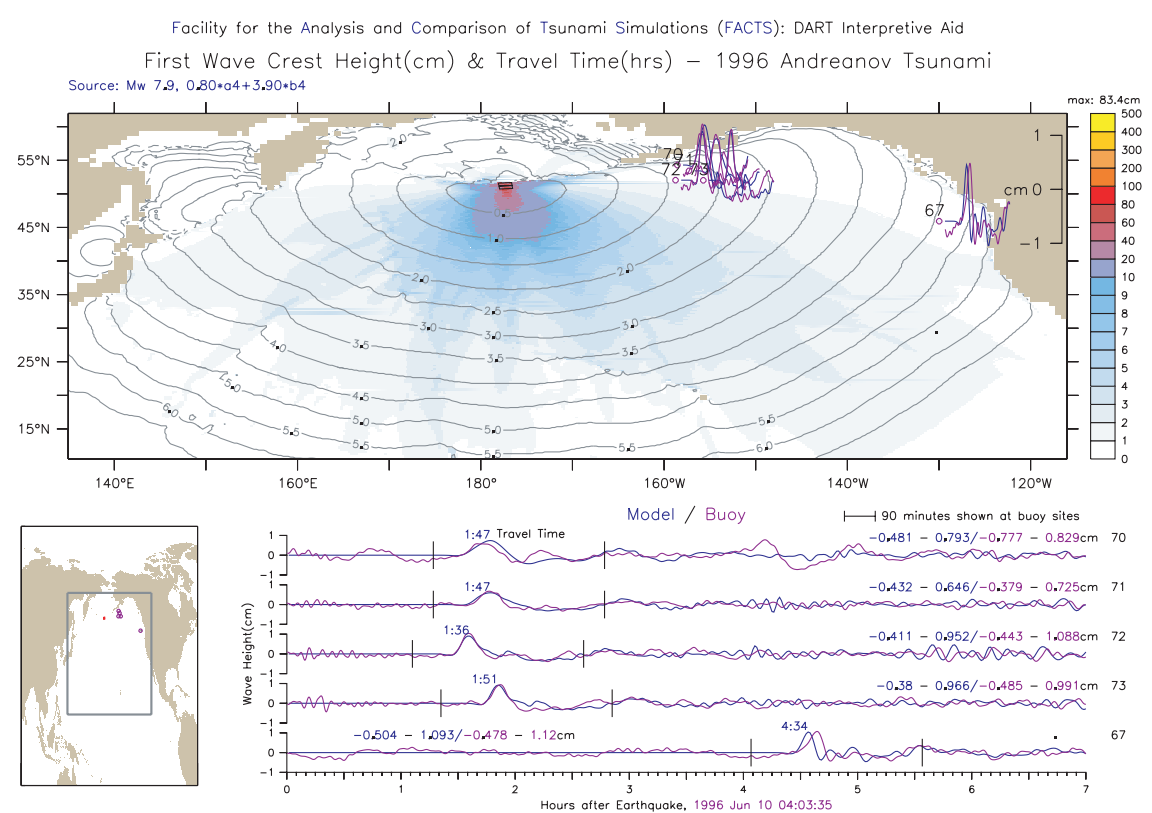

Figure 4. Results of BPR data inversion for 1996 Anderanov Island tsunami. Top frame shows the source inferred by the inversion (black rectangles show unit sources' fault plains), maximum computed amplitudes of tsunami from this source (filled colored contours), travel time contours in hours after earthquake (solid lines), and locations of the BPRs. Bottom frame shows a reference map (left) and comparison of the model (blue) and BPR data (magenta).

typical mechanism for the displacement). Therefore, a discrete set of unit sources (Green's functions) can provide the basis for constructing a tsunami scenario that would simulate a given tsunameter data. $\mathrm{Nu}-$ merical solutions of tsunami propagation from these unit sources, when linearly combined, provide arbitrary tsunami simulation for the data assimilation step of the forecast scheme.

This principle is used to construct a tsunami forecast database of pre-computed unit propagation solutions for sources around the North Pacific (Figure 5). All unit scenarios are thoroughly tested and scrutinized for reasonableness and sensitivity to errors. The database stores all simulation data for each unit solution, including amplitudes and velocities for each offshore location around the North Pacific. This approach allows the completion of data assimilation without additional time-consuming model runs. It also provides the offshore forecast of tsunami amplitudes and all other wave parameters around the North Pacific immediately after the data assimilation is complete. 


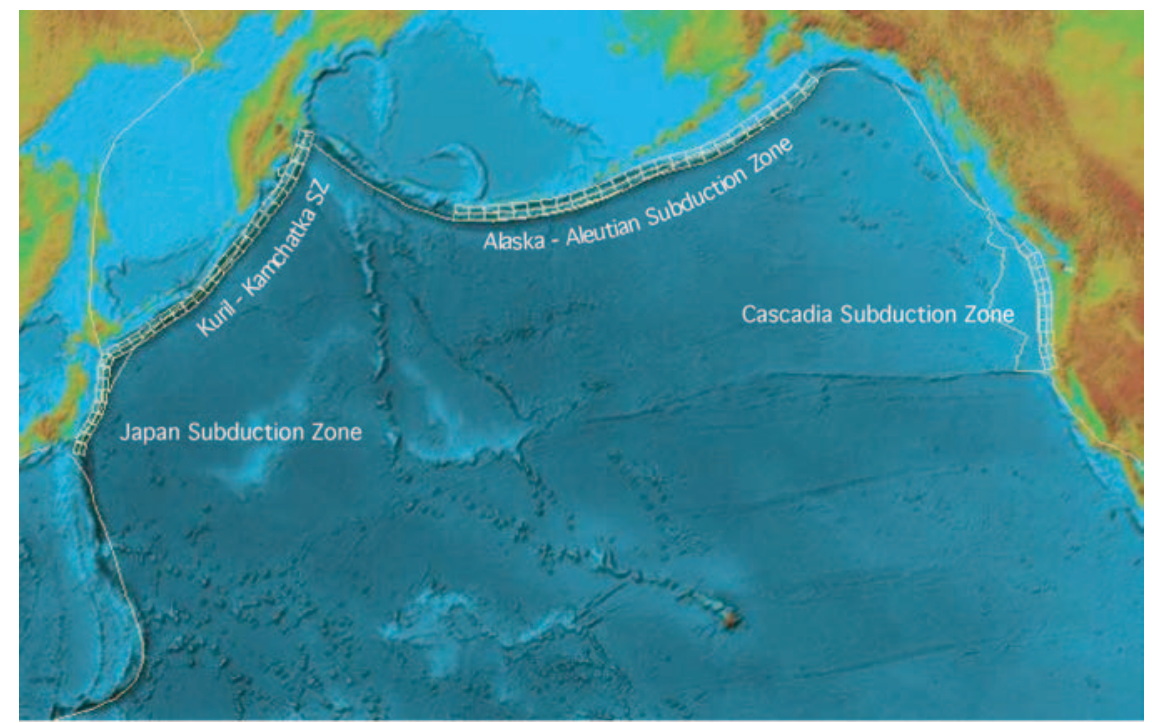

Figure 5. Input data for the offshore forecast model database. Bathymetric data for the database computation is shown as a shaded relief map. White rectangles show fault plains for the unit sources included in the database. Major plate boundaries are shown as white lines.

\subsection{Source CORRECTION USING tSunameter}

The previously described inversion algorithm is implemented to work with the forecast database. It combines real-time tsunameter data of offshore amplitude with the simulation database to improve accuracy of an initial offshore tsunami scenario.

\subsection{INUNDATION ESTIMATES WITH NON-LINEAR MODEL}

Once the offshore scenario is obtained, the results of the propagation run are used for the site-specific inundation forecast. Tsunami inundation is a highly nonlinear process. Therefore, linear combinations of different inundation runs cannot be combined to obtain a valid solution. A high-resolution 2-D inundation model is run to obtain a local inundation forecast. Data input for the inundation computations are the results of the offshore forecast - tsunami parameters along the perimeter of the inundation computation area. The forecast inundation model can be optimized to obtain local forecasts within minutes on modern computers. Nevertheless, obtaining inundation estimates for many communities at the same time can take too much time. We consider different approaches to reduce the inundation forecast time, including using parallel supercomputers and/or distributed computation of local inundation via a web interface. Simplified methods of inunda- 
tion estimation are also being considered for fast preliminary estimates of coastal amplitudes, such as one-dimensional runup estimates (one spatial dimension), analytical extrapolation of the offshore values to the coast, and others.

In summary, to forecast inundation by early tsunami waves, seismic parameter estimates and tsunami measurements are used to sift through a pre-computed generation/propagation forecast database and select an appropriate (linear) combination of scenarios that most closely matches the observational data. This produces estimates of tsunami characteristics in deep water which can then be used as initial conditions for a site-specific (non-linear) inundation algorithm. A statistical methodology has been developed to forecast the maximum height of later tsunami waves that can threaten rescue and recovery operations. The results are made available through a user-friendly interface to aid hazard assessment and decision making by emergency managers. The focus of this initial effort is on forecasting for tsunamis in the North Pacific. The MOST model performed computations of generation/propagation scenarios for the forecast database. The non-linear 2-dimensional high-resolution model provides the inundation forecasts.

\subsection{Live Access Server}

Live Access Server technology developed at PMEL (http://ferret. wrc.noaa.gov/Ferret/LAS/ferret_LAS.html) was used to build a Webbased user interface to exploit the database approach for the offshore forecast. The Web interface allows platform-independent access to the forecasting tools by emergency managers worldwide.

The interface allows creation of a variety of offshore tsunami propagation scenarios by combining pre-computed propagation solutions. It also provides access to the real-time tsunameter data and some historical deep-ocean BPR data. The built-in data inversion scheme combines the deep-ocean data with the simulation database to automatically determine the best tsunami scenario that corresponds to tsunami measurements.

\section{Testing Tsunami Forecasting Methodology}

The limited amount of historical deep-ocean tsunami records does not include tsunamis that have been destructive or caused inundation to the U.S. coasts. However, there are several events that were recorded by both deep-ocean and coastal gages. The SIFT methodology has been tested against two such tsunamis: the 10 June 1996 Andreanov Is. (Tanioka and González, 1998) and the 4 October 1994 Kuril Is. (Yeh et al., 

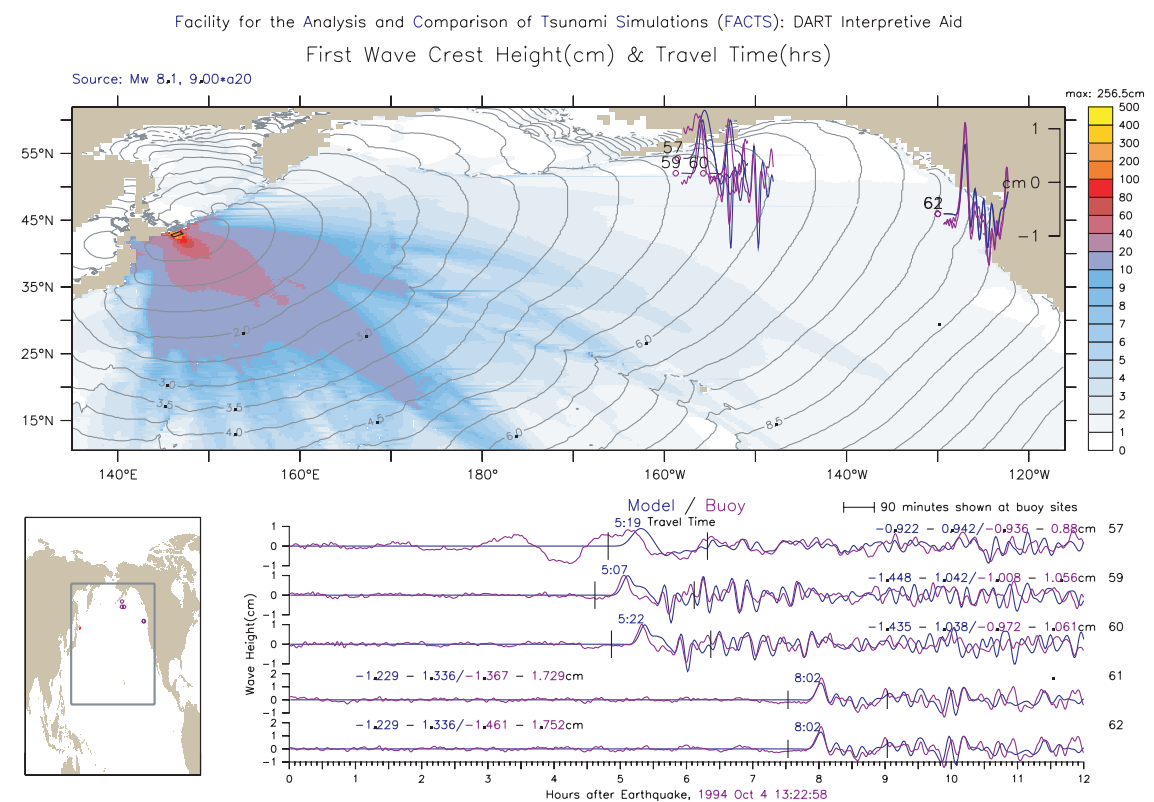

Figure 6. Offshore forecast for the 1994 Kuril Island tsunami. Notations are the same as in Figure 4.

1995) events. Both events were recorded by several BPRs at similar locations offshore of Alaska and the U.S. West Coast. The coastal tide gage record of these events at Hilo, HI, was used for comparison with the coastal forecast.

The offshore model scenario for the Andreanov event was obtained from the forecast database by inverting data from just one BPR as described earlier (Figure 4). The inversion of the Kuril data was done using all five BPR recordings; the results are shown in Figure 6. These model scenarios were then used as input for the high-resolution inundation model for Hilo Bay. The model computed tsunami dynamics on several nested grids, with the highest spatial resolution of 30 meters inside Hilo Bay (Figure 7). Neither tsunami produced inundation at Hilo, but both recorded nearly half a meter signal at the Hilo gage. Model forecast predictions for this tide gage are compared with observed data in Figure 7. The comparison demonstrates that amplitudes, arrival time, and periods of several first waves of the tsunami wave train were forecasted correctly. More tests are required to ensure that the inundation forecast would work for arbitrary tsunamis. Nevertheless, these first tests indicate that the methodology for tsunami forecast works and useful tools could be developed and implemented soon. 


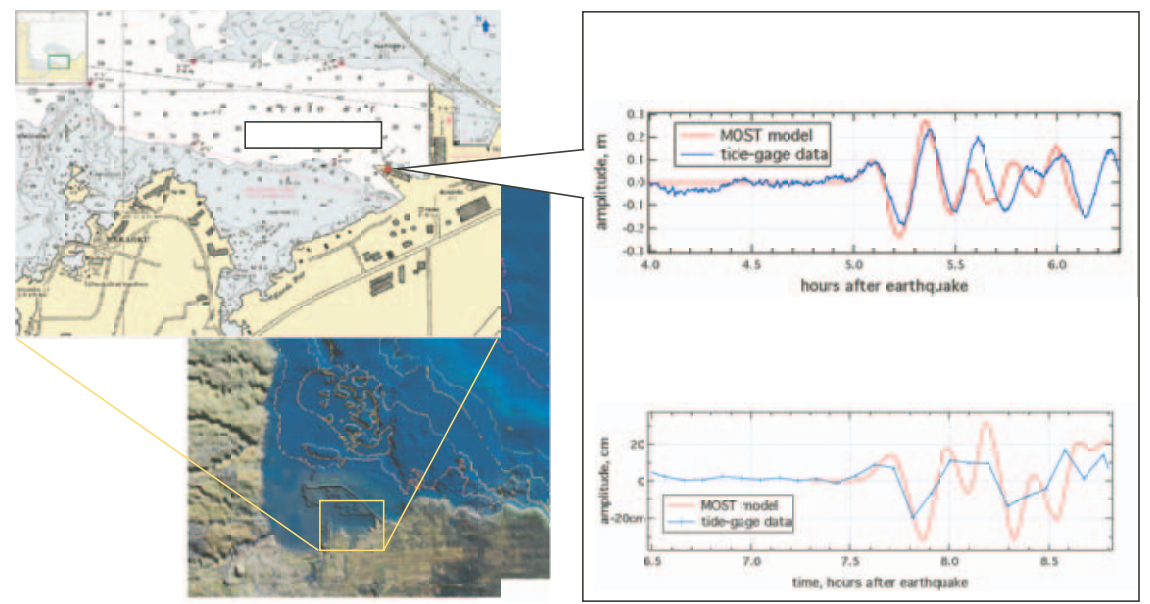

Figure 7. 7. Coastal forecast at Hilo, HI for 1996 Andreanov Is. (top) and 1994 Kuril Is. (bottom) tsunamis. Left frame shows location of Hilo tide-gage (top map) and digital elevation data for the high-resolution inundation computation (bottom map). Right frame shows comparison of the forecasted (red line) and measured (blue line) gage data.

\section{Summary}

This article describes tsunami forecasting methodology and prototype modeling tools developed by the Pacific Marine Environmental Laboratory of the National Oceanic and Atmospheric Administration. The methodology will be the foundation of the next generation forecast tools for tsunami warning and mitigation that are being developed in close collaboration with Tsunami Warning Centers and academia. The new tools will provide site- and event-specific forecast of tsunami amplitudes for the entire Pacific to assist emergency managers during tsunami warning and mitigation procedures.

\section{Acknowledgements}

Acknowledgments go here.

\section{References}

Bourgeois, J., C. Petroff, H. Yeh, V. Titov, C. Synolakis, B. Benson, J. Kuroiwa, J. Lander, and E. Norabuena: 1999, 'Geologic setting, field survey and modeling of the Chimbote, northern Peru, tsunami of 21 February 1996'. Pure Appl. Geophys. 154(3/4), 513-540. 
González, F., D. Burwell, K. F. Cheung, C. McCreery, H. Mofjeld, V. Titov, and P. Whitmore: 2003, 'Far-field Tsunami Forecast Guidance'. NOAA Tech. Memo., NOAA/Pacific Marine Environmental Laboratory, Seattle, WA.

González, F. I. et al.: this issue, 'Tsunameter paper'. Nat. Hazards.

Mofjeld, H. O., F. I. González, E. N. Bernard, and J. C. Newman: 2000, 'Forecasting the heights of later waves in Pacific-wide tsunamis'. Nat. Hazards 22, 71-89.

Takahashi, T.: 1996, 'Benchmark Problem 4. The 1993 Okushiri tsunami-Data, Conditions and Phenomena'. In: H. Yeh, P. Liu, and C. Synolakis (eds.): Long wave runup models. Singapore: World Scientific Publishing Co. Pte. Ltd., pp. 384-403.

Tanioka, Y. and F. I. González: 1998, 'The Aleutian earthquake of June 10, 1996 (Mw 7.9) ruptured parts of both the Andreanov and Delarof segments'. Geophys. Res. Lett. 25(12), 2245-2248.

Titov, V. and F. I. González: 1997, 'Implementation and testing of the Method of Splitting Tsunami (MOST) model'. Technical Report NOAA Tech. Memo. ERL PMEL-112 (PB98-122773), NOAA/Pacific Marine Environmental Laboratory, Seattle, WA.

Titov, V. V., H. O. Mofjeld, F. I. González, and J. C. Newman: 1999, 'Offshore forecasting of Alaska-Aleutian Subduction Zone tsunamis in Hawaii'. NOAA Tech. Memo ERL PMEL-114, NOAA/Pacific Marine Environmental Laboratory, Seattle, WA.

Titov, V. V., H. O. Mofjeld, F. I. González, and J. C. Newman: 2001, 'Offshore forecasting of Alaskan tsunamis in Hawaii'. In: G. T. Hebenstreit (ed.): Tsunami Research at the End of a Critical Decade. Birmingham, England: Kluwer Acad. Pub., Netherlands, pp. 75-90.

Titov, V. V. and C. E. Synolakis: 1995, 'Modeling of breaking and nonbreaking long wave evolution and runup using VTCS-2'. J. Waterw. Port Coast. Ocean Eng. 121(6), 308-316.

Titov, V. V. and C. E. Synolakis: 1996, 'Numerical modeling of 3-D long wave runup using VTCS-3'. In: H. Yeh, P. Liu, and C. Synolakis (eds.): Long wave runup models. Singapore: World Scientific Publishing Co. Pte. Ltd., pp. 242-248.

Titov, V. V. and C. E. Synolakis: 1997, 'Extreme inundation flows during the Hokkaido-Nansei-Oki tsunami'. Geophys. Res. Lett. 24(11), 1315-1318.

Titov, V. V. and C. E. Synolakis: 1998, 'Numerical modeling of tidal wave runup'. J. Waterw. Port Coast. Ocean Eng. 124(4), 157-171.

Wei, Y., K. F. Cheung, G. D. Curtis, and C. S. McCreery: 2003, 'Inverse algorithm for tsunami forecasts'. J. Waterw. Port Coast. Ocean Eng. 129(3), 60-69.

Whitmore, P. M.: 2003, 'Tsunami Amplitude Prediction During Events: A Test Based on Previous Tsunamis'. In: Science of Tsunami Hazards, Vol. 21. pp. $135-143$.

Yeh, H., V. V. Titov, V. Gusyiakov, E. Pelinovsky, V. Khramushin, and V. Kaistrenko: 1995, 'The 1994 Shikotan earthquake tsunami'. Pure Appl. Geophys. 144(3/4), 569-593. 